\title{
Multiplicity spectra in pp collisions at high energies in terms of Gamma and Tsallis distributions
}

\author{
S. Sharma and M. Kaur \\ Department of Physics, Panjab University, Chandigarh 160 014, India
}

(Received 16 February 2018; published 7 August 2018)

\begin{abstract}
In recent years the Tsallis statistics is gaining popularity in describing charged particle production and their properties, in particular $p_{T}$ spectra and the multiplicities in high energy particle collisions. Motivated by its success, an analysis of the LHC data of proton-proton collisions at energies ranging from 0.9 to $7 \mathrm{TeV}$ in different rapidity windows for charged particle multiplicities has been done. A comparative analysis is performed in terms of the Tsallis distribution, the Gamma distribution and the shifted-Gamma distribution. An interesting observation on the inapplicability of these distributions at $\sqrt{s}=7 \mathrm{TeV}$ in the lower rapidity windows is intriguing. The nonextensive nature of the Tsallis statistics is studied by determining the entropic index and its energy dependence. A two-component approach in terms of fractions of events without minijets and events with minijets is applied to improve the fittings. The analysis is extrapolated to predict the multiplicity distribution at $\sqrt{s}=14 \mathrm{TeV}$ for one rapidity window, $|y|<1.5$ with the Tsallis function.
\end{abstract}

DOI: 10.1103/PhysRevD.98.034008

\section{INTRODUCTION}

In the high energy collisions of particles, several new particles are produced. Experiments study the particle properties averaged over multiple collisions. It is well known that properties such as the mean multiplicities, transverse momenta etc. follow rules of statistical mechanics. Amongst the models of statistical particle production, the Tsallis distribution [1,2] has been used extensively to describe transverse momentum spectra of particles stemming from high energy particle collisions. Both for intermediate and high momenta regions, the particle production has been successfully described in the proton-proton $(p p)$, antiprotonproton $(\bar{p} p)$, nucleus-nucleus $(A A)$ [3-7] and $e^{+} e^{-}[8,9]$ collisions. It has also been shown by Urmossy et al. [10] that in very high energy collisions, if only the momentum-energy conservation in hadronization is taken into account, the average momentum distribution of produced particles can be considered as a microcanonical generalization of the Tsallis distribution. Each final state particle created in a collision is identified with a microstate of a microcanonical ensemble with scaling volume fluctuations. If the momentum distribution in events with fixed multiplicity is microcanonical, the shifted multiplicity has the Gamma-distribution. In the Tsallis $q$-statistics, the entropy of standard statistical

\footnotetext{
"manjit@pu.ac.in
}

Published by the American Physical Society under the terms of the Creative Commons Attribution 4.0 International license. Further distribution of this work must maintain attribution to the author(s) and the published article's title, journal citation, and DOI. Funded by SCOAP . mechanics becomes nonextensive. This nonextensive property of the entropy is then determined in terms of a parameter $q$, known as entropic index, which on account of its nonextensive behavior exceeds unity. Most of the analyses on the data from different kinds of collisions have been done to study the transverse momentum distributions and fragmentation functions. At LHC energies, some analyses [11-14] have been done to study the $p_{T}$ spectra by using the Tsallis distribution. However, the analysis of the multiplicities has been done only in very few cases [15-17] using different approaches. In addition, analyses of the data from various experiments at the RHIC, STAR, PHENIX, ATLAS and CMS collaborations have shown excellent fits to the transverse momentum distributions with the Tsallis-like distribution [18]. The charged hadron yields as a function of the transverse momentum at four different energies from the ATLAS [19] collaboration and the UA1 [20] collaboration have been analyzed by Cleymans et al. [18]. A good description by the Tsallis distribution makes it applicable for hard QCD processes. Authors interpret this as a possible manifestation of the duality between the statistical and dynamical description of strong interactions [21]. In another analysis, Capella et al. [22] have studied the multiplicities at the LHC energies in the Pomeron model.

In this paper, the first study of multiplicity distributions is reported on the proton-proton collisions at the LHC energies in the restricted central rapidity windows. Energymomentum conservation strongly influences the multiplicity distribution for the full phase space. The distribution in restricted rapidity windows, however, is less prone to such constraints and thus can be expected to be a more sensitive probe to the underlying dynamics of QCD, as inferred in 

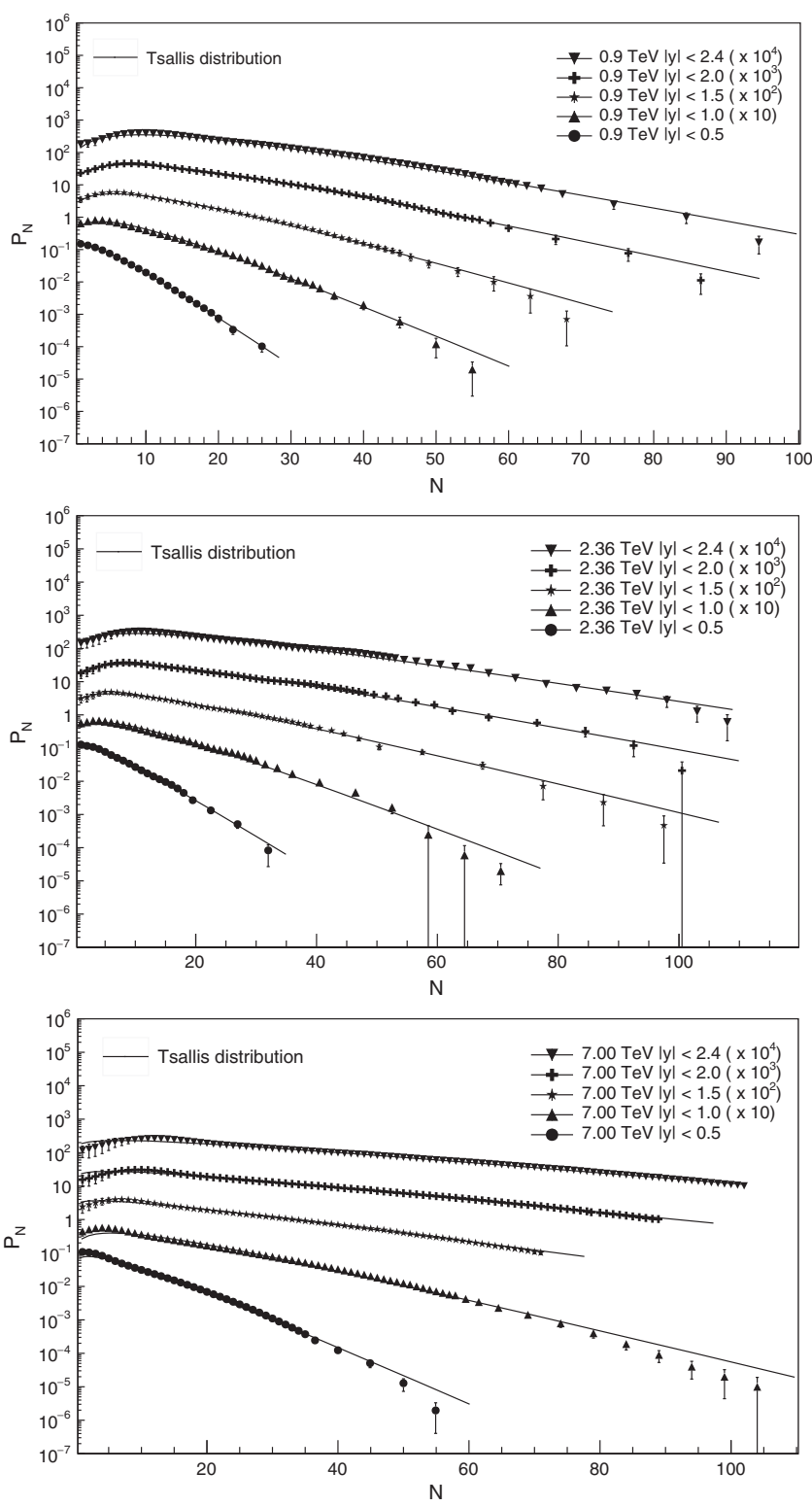

FIG. 1. The charged particle multiplicity distributions measured in $p p$ collisions by the CMS experiment with the fits by the Tsallis distribution.

Refs. [10,23]. We also study the dependence of the entropic index $q$, an important parameter in the Tsallis $q$-statistics, on energy of the collisions.

After this brief introduction in Sec. I, we describe the essential steps of the distributions, Tsallis, Gamma and shifted-Gamma and the basic definition of rapidity used, in Sec. II. Section III gives details of the data used and results from the comparison of our analysis of the three distributions. Section IV presents the conclusions.

\section{PARTICLE PRODUCTION AND THE DISTRIBUTION}

The charged particles produced in a collision are emitted at all angles and measured in terms of rapidity defined as
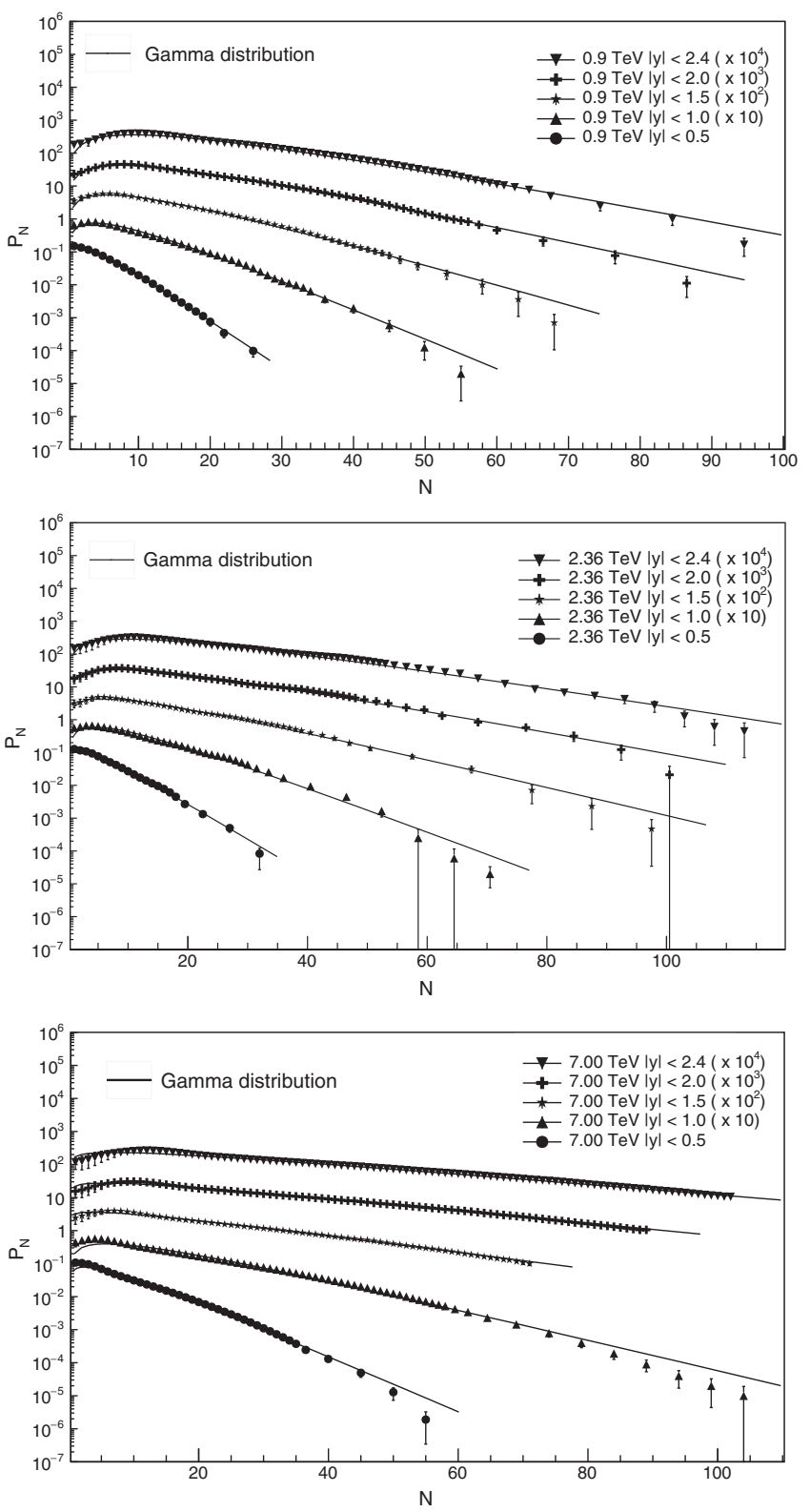

FIG. 2. The charged particle multiplicity distributions measured in $p p$ collisions by the CMS experiment with the fits by the Gamma distribution.

$y=\frac{1}{2} \ln \left(\frac{E+p_{L}}{E-p_{L}}\right)$, where $\mathrm{E}$ is the particle energy and $p_{L}$ is the longitudinal momentum. The number of particles produced is distributed according to some probability distribution function (PDF) with mean of the distribution coinciding with the average number, called the average multiplicity. We discuss three such PDFs in the following section.

\section{A. The Gamma and the shifted-Gamma distributions}

The Gamma is a very basic distribution which describes the multiplicity distributions at lower energies very well. Inclusive data $e^{+} e^{-} \rightarrow h^{ \pm}+X$ from LEP experiments were studied by Urmossy et al. [10] by considering a 

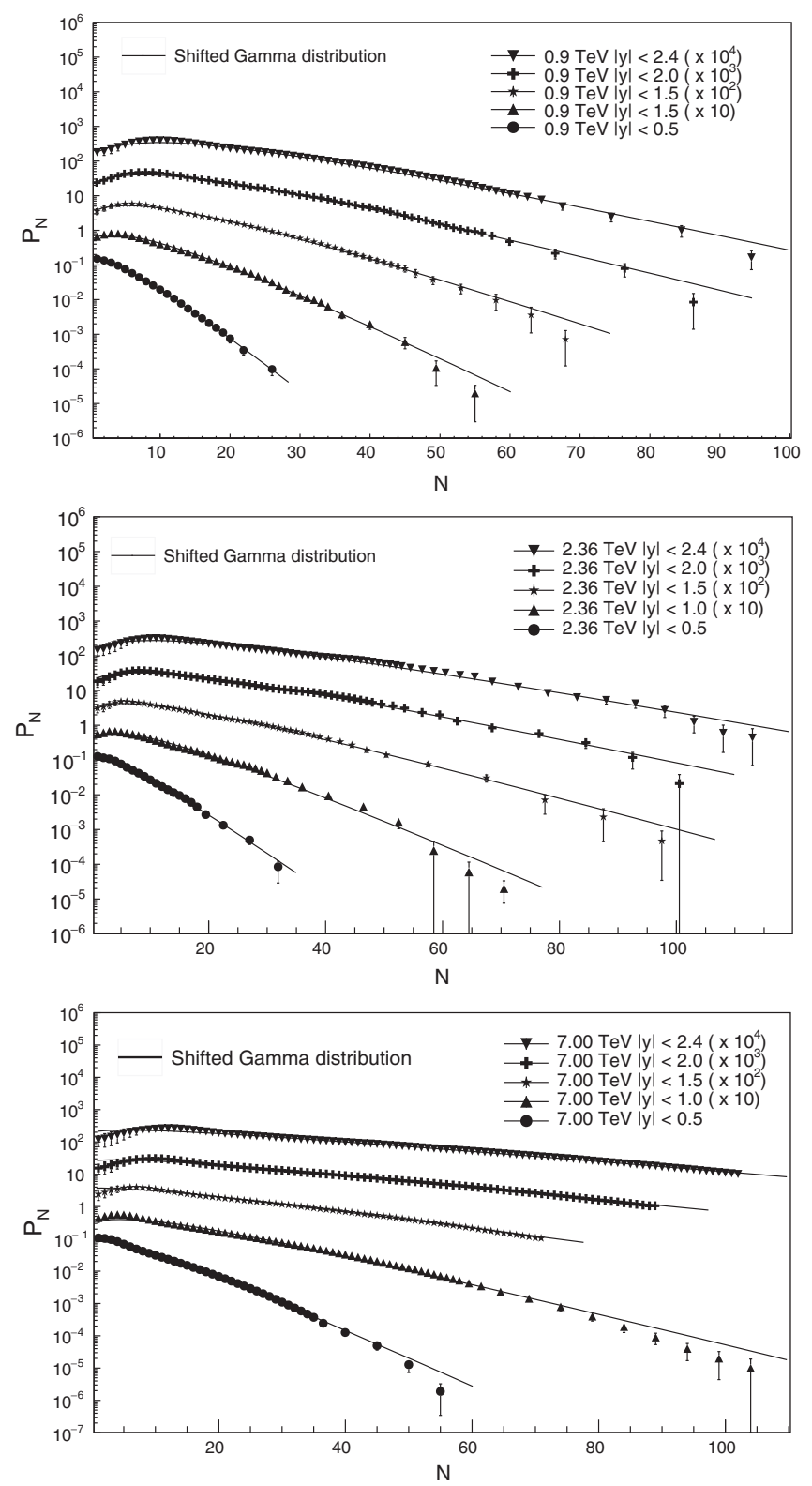

FIG. 3. The charged particle multiplicity distributions measured in $p p$ collisions by the CMS experiment with the fits by the shifted-Gamma distribution.

sample of two-jet events. They used the Boltzmann-Gibbs and microcanonical distributions in one dimension. They showed that the Gamma distribution of the shifted multiplicity, $N-N 0$, can result in a Tsallis or microcanonical Tsallis shaped spectrum.

The probability density function for the Gamma and the shifted-Gamma distributions are given below:

$$
P_{N}=A N^{\alpha-1} \exp ^{-\beta N}
$$

with $\alpha$ the scale parameter, $\beta$ the shape parameter and $A$ are the fit parameters of the distribution. The average momentum distribution is Tsallis.
A shift in the multiplicity $N \rightarrow(N-N 0)$ is exploited, without violating the Koba-Nielsen-Olesen (KNO) scaling [24], then the averaging is done over the multiplicity distribution,

$$
P_{N}=A(N-N 0)^{\alpha^{\prime}-1} \exp ^{-\beta^{\prime}(N-N 0)} .
$$

The resulting momentum distribution is a possible microcanonical generalization of the Tsallis. The shift in the multiplicity has been chosen to be $N 0=1+2 / D$ where D is the dimensionality of phase space. The details can be found in $[10,23]$.

\section{B. The Tsallis distribution}

The Tsallis $q$-statistics deals with entropy in the usual Boltzmann-Gibbs thermostatistics modified by introducing the $q$-parameter. For a given thermodynamical system, when divided into two subsystems, the Tsallis entropy no longer remains extensive, but is defined as

$$
S_{q}(A, B)=S_{A}+S_{B}+(1-q) S_{A} S_{B},
$$

where $q$ is known as the entropic index with value $q>1$ and $(1-q)$ measures the departure of entropy from its extensive behavior. Assuming the interaction as a canonical ensemble of $N$ particles, the partition function is defined through the probability as

$$
P_{N}=\frac{Z_{q}^{N}}{Z}
$$

where $Z$ represents the total partition function and $Z_{q}^{N}$ represents partition function at a particular multiplicity. Aguiar et al. [2] have discussed in detail the method for calculating the $N$ particles partition function and deriving the probability distribution. Details of these calculations can be obtained from this reference.

\section{RESULTS}

The experimental data of proton-proton collisions at the Large Hadron Collider (LHC) obtained by the CMS experiment for different energies are analyzed. The data analyzed are at $\sqrt{s}=0.9,2.34$ and $7 \mathrm{TeV}$ in the restricted rapidity windows of $|y|<0.5,1.0,1.5,2.0$ and 2.4. The experimental data [25] are fitted with the distributions from the Tsallis $q$-statistics, the Gamma distribution and the shifted-Gamma distribution. The results are discussed in the following sections.

\section{A. The Gamma versus the Tsallis distribution}

The probabilities from the Gamma distribution, the shifted-Gamma distribution and the Tsallis distribution are calculated using Eqs. (1), (2) and (4). Fits to the data are shown in Figs. 1-3. Table I gives the parameters of the 
TABLE I. Fit parameters of the distributions for all rapidity windows for the $p p$ data.

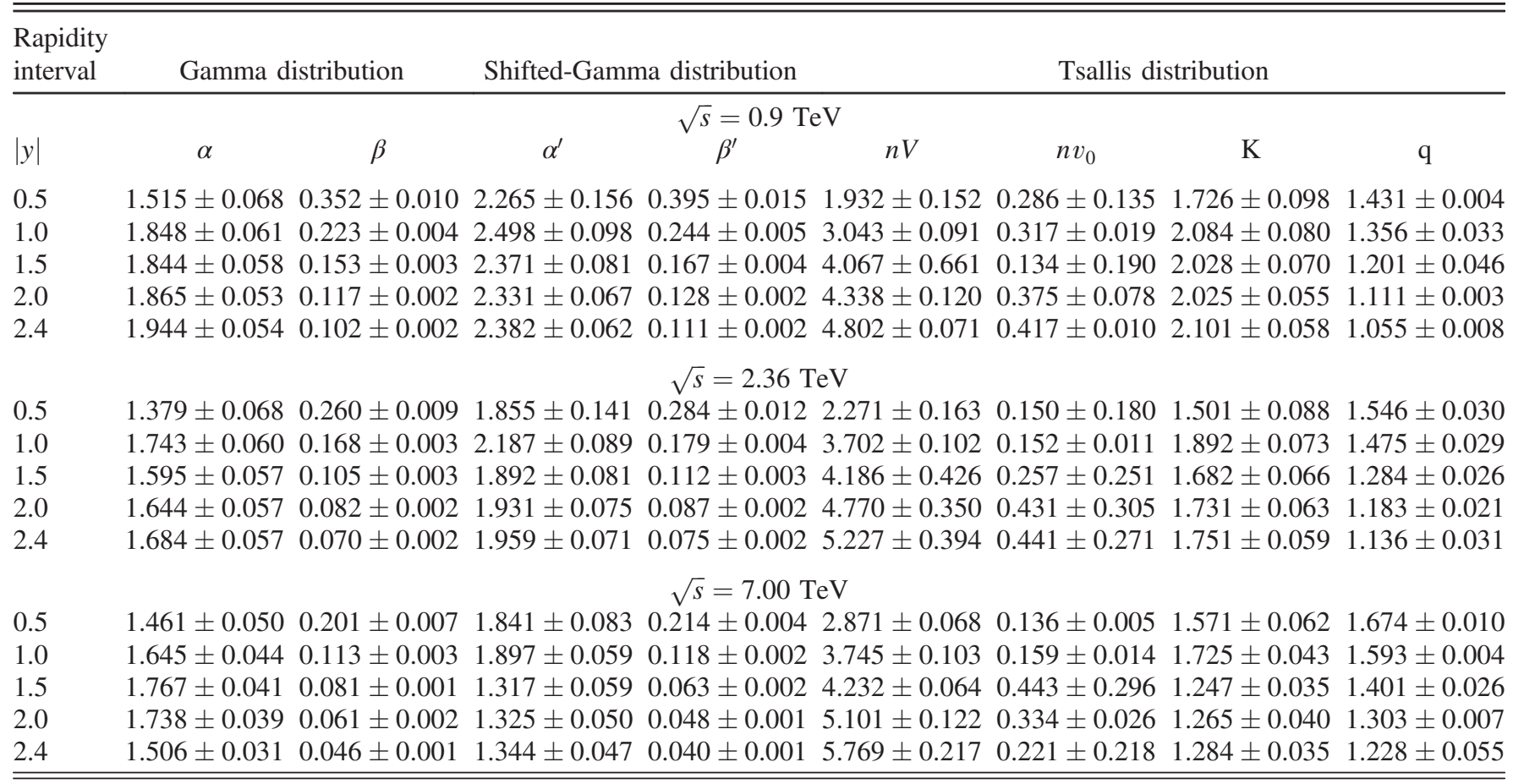

fits for all the rapidity windows at energies $0.9,2.36$ and $7 \mathrm{TeV}$ respectively and a comparison of corresponding $\chi^{2} /$ ndf and $p$-values are given in Table II. While fitting we consider the probability distribution for $7 \mathrm{TeV}$ extending up to the continuous range of $N$ values. Beyond this the statistics is very low and the probability falls below 0.001 leading to the fit parameters with very large errors, particularly for the Tsallis distribution.

In order to study the behavior of the three distributions for multiplicities of charged particles produced with higher transverse momenta $p_{T}$, the analysis is extended to the data $\sqrt{s}=0.9,2.36$ and $7 \mathrm{TeV}$ in the restricted rapidity window of $|y|<2.4$ and $p_{T}>500 \mathrm{MeV}$. Figure 4 shows the results of fits for the three distributions. The fit parameters are given in Table III and the $\chi^{2} /$ ndf and $p$-values in Table IV.

One finds that both the Tsallis and the shifted-Gamma distributions reproduce the data very well in most of the rapidity windows at the three energies in comparison to the Gamma distribution. However all the distributions fail for rapidity windows $|y|<0.5$ and $|y|<1.0$ with $p$-values

TABLE II. $\chi^{2} /$ ndf comparison for the fits with three different distributions for all rapidity windows for the $p p$ data.

\begin{tabular}{|c|c|c|c|c|c|c|c|}
\hline \multirow[b]{2}{*}{ Energy $(\mathrm{TeV})$} & \multirow{2}{*}{$\frac{\text { Rapidity interval }}{|y|}$} & \multicolumn{2}{|c|}{ Gamma distribution } & \multicolumn{2}{|c|}{ Shifted-Gamma distribution } & \multicolumn{2}{|c|}{ Tsallis distribution } \\
\hline & & $\chi^{2} / \mathrm{ndf}$ & $p$ value & $\chi^{2} / \mathrm{ndf}$ & $p$ value & $\chi^{2} / \mathrm{ndf}$ & $p$ value \\
\hline \multirow[t]{5}{*}{0.9} & 0.5 & $3.21 / 19$ & 1.0000 & $0.98 / 19$ & 1.0000 & $1.58 / 17$ & 1.0000 \\
\hline & 1.0 & $54.50 / 36$ & 0.0247 & $33.66 / 36$ & 0.5804 & $43.64 / 34$ & 0.1244 \\
\hline & 1.5 & $48.49 / 48$ & 0.4531 & $35.41 / 48$ & 0.9113 & $41.32 / 46$ & 0.6683 \\
\hline & 2.0 & $38.11 / 58$ & 0.9798 & $31.21 / 58$ & 0.9985 & $33.06 / 56$ & 0.9938 \\
\hline & 2.4 & $52.93 / 64$ & 0.8368 & $44.02 / 64$ & 0.9733 & $46.82 / 62$ & 0.9240 \\
\hline \multirow[t]{5}{*}{2.36} & 0.5 & $7.41 / 19$ & 0.9917 & $5.94 / 19$ & 1.0000 & $6.60 / 17$ & 0.9882 \\
\hline & 1.0 & $67.79 / 36$ & 0.0011 & $50.59 / 36$ & 0.0541 & $59.99 / 34$ & 0.0039 \\
\hline & 1.5 & $30.10 / 46$ & 0.9662 & $25.24 / 46$ & 0.9945 & $27.28 / 44$ & 0.9774 \\
\hline & 2.0 & $46.44 / 56$ & 0.8162 & $45.25 / 56$ & 0.8473 & $45.31 / 54$ & 0.7941 \\
\hline & 2.4 & $44.91 / 65$ & 0.9729 & $44.98 / 65$ & 0.9778 & $40.94 / 63$ & 0.9859 \\
\hline \multirow[t]{5}{*}{7.00} & 0.5 & $101.40 / 37$ & 0.0001 & $75.86 / 37$ & 0.0002 & $91.74 / 35$ & 0.0001 \\
\hline & 1.0 & $183.71 / 66$ & 0.0001 & $149.11 / 66$ & 0.0001 & $170.93 / 64$ & 0.0001 \\
\hline & 1.5 & $34.93 / 68$ & 0.9997 & $35.89 / 68$ & 0.9995 & $35.38 / 66$ & 0.9991 \\
\hline & 2.0 & $40.41 / 86$ & 1.0000 & $44.27 / 86$ & 0.9999 & $41.69 / 84$ & 1.0000 \\
\hline & 2.4 & $47.91 / 99$ & 1.0000 & $54.67 / 99$ & 0.9999 & $49.96 / 97$ & 1.0000 \\
\hline
\end{tabular}



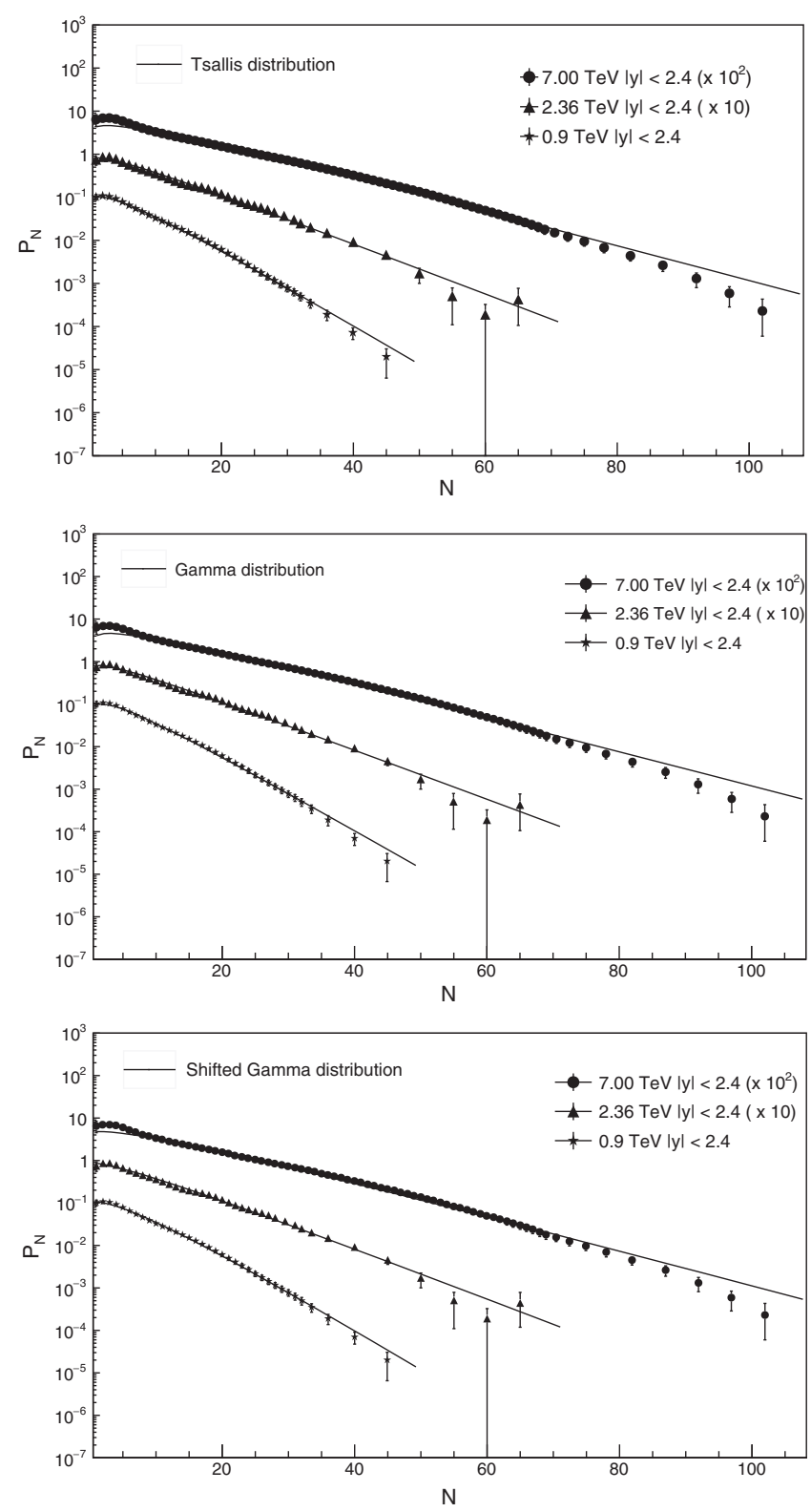

FIG. 4. The charged multiplicity distributions for particles with $|y|<2.4, p_{T}>500 \mathrm{MeV}$ measured by the CMS experiment with the fits by the Tsallis, the Gamma and the shifted-Gamma distributions. corresponding to $\mathrm{CL}<0.10 \%$. The detailed comparison between the three functions is shown in Table II where $\chi^{2} /$ ndf and $p$-values at all energies for all rapidity windows are compared. It is found that the $\chi^{2} / \mathrm{ndf}$ values are comparable for the Tsallis and the shifted-Gamma fits with $p$-values corresponding to $\mathrm{CL}>0.1 \%$ as compared to the Gamma fits. This is true for nearly all the rapidity windows at all the energies.

Results from Tables III and IV for the fits of the three distributions to the data with $p_{T}>500 \mathrm{MeV}$ in the $|y|<$ 2.4 rapidity window show that for $7 \mathrm{TeV}$ data $p$-values correspond to $\mathrm{CL}<0.1 \%$ and hence all the fits are statistically excluded at this energy. However at the other two energies, all fits are able to reproduce the data, with the shifted-Gamma giving the best result.

From Tables I and III, it is also observed that for the Gamma distribution, $\beta$ values decrease with energy as well as with rapidity. This is as per the expected trend. Similarly for the shifted-Gamma distribution, $\beta^{\prime}$ values decrease with energy as well as with rapidity. Both $\beta$ and $\beta^{\prime}$ measure the shape parameters for the two distributions. For the Tsallis distribution, the $q$ value which measures the entropic index of the Tsallis statistics, increases with energy and exceeds unity in each case. This confirms that the Tsallis statistics becomes nonextensive. The parameter $K$ also determines the shape of the distribution, it becomes binomial-like if $K$ becomes negative.

Figure 5 shows energy dependence of the entropic parameter of the Tsallis function in various rapidity windows. The dependence can be parametrized as a power law, $q=A \sqrt{s}^{B}$. The fit parameters $A$ and $B$ are listed in Table V. It may be observed that the value of $B$ varies very slightly, taking into account the errors on $B$ with rapidity. In a study of the systematic properties of Tsallis distribution, Cleyman et al. [18] have studied the energy dependence of parameters of Tsallis distribution in $p p$ collisions and shown that $q$ has a weak dependence on beam energy. From the transverse momentum distributions, they have determined the $q$ values from the ATLAS data [19] at $\sqrt{s}=0.9$, 2.36 and $7 \mathrm{TeV}$ as $1.1217 \pm 0.0007,1.1419 \pm 0.0025$ and $1.1479 \pm 0.0008$. These values agree very closely with the values we obtain from multiplicity distribution fits in

TABLE III. Fit parameters with the distributions for charged particle multiplicity spectra in $|y|<2.4$ and $P_{T}>500 \mathrm{MeV}$ of the $p p$ data.

\begin{tabular}{|c|c|c|c|c|c|c|c|c|}
\hline \multirow[b]{2}{*}{$\begin{array}{l}\text { Energy } \\
(\mathrm{TeV})\end{array}$} & \multicolumn{2}{|c|}{ Gamma distribution } & \multicolumn{2}{|c|}{ Shifted-Gamma distribution } & \multicolumn{4}{|c|}{ Tsallis distribution } \\
\hline & $\alpha$ & $\beta$ & $\alpha^{\prime}$ & $\beta^{\prime}$ & $n V$ & $n v_{0}$ & $\mathrm{~K}$ & $\mathrm{q}$ \\
\hline 0.9 & $1.386 \pm 0.057$ & $0.211 \pm 0.005$ & $1.782 \pm 0.098$ & $0.227 \pm 0.006$ & $2.214 \pm 0.054$ & $0.249 \pm 0.054$ & $1.476 \pm 0.056$ & $1.055 \pm 0.036$ \\
\hline 2.36 & $1.204 \pm 0.054$ & $0.137 \pm 0.003$ & $1.373 \pm 0.093$ & $0.142 \pm 0.005$ & $2.602 \pm 0.106$ & $0.282 \pm 0.028$ & $1.241 \pm 0.063$ & $1.128 \pm 0.007$ \\
\hline 7.00 & $1.288 \pm 0.037$ & $0.096 \pm 0.001$ & $1.438 \pm 0.048$ & $0.099 \pm 0.001$ & $3.611 \pm 0.110$ & $0.177 \pm 0.093$ & $1.326 \pm 0.033$ & $1.242 \pm 0.092$ \\
\hline
\end{tabular}


TABLE IV. $\chi^{2} /$ ndf values obtained with the distributions fits to the charged particle multiplicity spectrum for $|y|<2.4$ and $P_{T}>$ $500 \mathrm{MeV}$ in the $p p$ data.

\begin{tabular}{|c|c|c|c|c|c|c|}
\hline \multirow[b]{2}{*}{ Energy $(\mathrm{TeV})$} & \multicolumn{2}{|c|}{ Gamma distribution } & \multicolumn{2}{|c|}{ Shifted-Gamma distribution } & \multicolumn{2}{|c|}{ Tsallis distribution } \\
\hline & $\chi^{2} / \mathrm{ndf}$ & $\overline{p \text { value }}$ & $\chi^{2} / \mathrm{ndf}$ & $p$ value & $\chi^{2} / \mathrm{ndf}$ & $p$ value \\
\hline 0.9 & $32.84 / 34$ & 0.5244 & $17.86 / 34$ & 0.9896 & $21.26 / 32$ & 0.9177 \\
\hline 2.36 & $36.37 / 36$ & 0.4514 & $33.43 / 36$ & 0.5914 & $35.61 / 34$ & 0.3195 \\
\hline 7.00 & $178.07 / 75$ & 0.0001 & $157.02 / 75$ & 0.0001 & $172.01 / 73$ & 0.0001 \\
\hline
\end{tabular}

rapidity $|y|<2.4$ for the CMS data; $1.055 \pm 0.008$, $1.136 \pm 0.031$ and $1.228 \pm 0.055$. Small differences are expected from the slightly different phase spaces considered in the two cases. Using the energy dependence of the Tsallis q parameter, the multiplicity distribution of charged particles is predicted in the $p p$ collisions in the restricted rapidity window of $|y|<1.5$ at $\sqrt{s}=14 \mathrm{TeV}$, as shown in Fig. 6. The value of $q$ is predicted as $1.476 \pm 0.108$. Similar predictions can be made for other higher rapidity windows. A consistent picture emerges in comparison to the results from other studies as mentioned.

In a further investigation of the failure of all distributions at $7 \mathrm{TeV}$, we consider the two-component approach, soft and semihard component structure in the multiparticle production. This leads to the division of the distribution in terms of soft events (events without minijets) and the semihard events (events with minijets). A distribution is then produced as a weighted superposition of the two components, the weight $\alpha_{\text {soft }}$ being the fraction of soft events, as below:

$$
P(n)=\alpha_{\text {soft }} P_{\text {soft }}^{\mathrm{MD}}(n)+\left(1-\alpha_{\text {soft }}\right) P_{\text {semi-hard }}^{\mathrm{MD}}(n) .
$$

The multiplicity distribution (MD) of each component is one of the three distributions under consideration; Gamma, shifted-Gamma or Tsallis distribution. The idea of this superposition was first suggested by Fuglesang [26] in order to explain the negative binomial regularity violations.

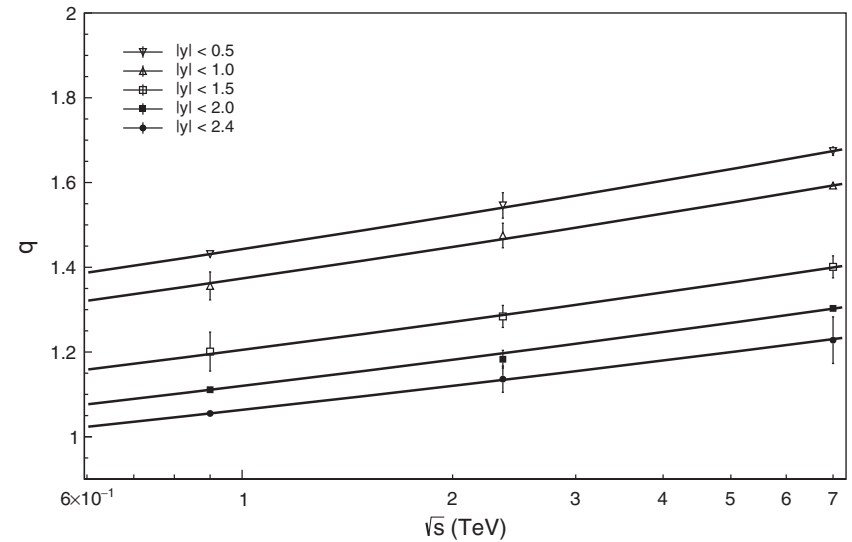

FIG. 5. The energy dependence of the nonextensive entropic parameter of the Tsallis function in different rapidity windows.
TABLE V. Parameters A and B of the power law fit between $\mathrm{q}$ and c.m. energy for the $p p$ collision data.

\begin{tabular}{lcc}
\hline \hline$|y|$ & $\mathrm{A}$ & $\mathrm{B}$ \\
\hline 0.5 & $0.851 \pm 0.020$ & $0.076 \pm 0.003$ \\
1.0 & $0.811 \pm 0.072$ & $0.076 \pm 0.010$ \\
1.5 & $0.706 \pm 0.106$ & $0.077 \pm 0.018$ \\
2.0 & $0.660 \pm 0.014$ & $0.076 \pm 0.003$ \\
2.4 & $0.630 \pm 0.081$ & $0.075 \pm 0.012$ \\
\hline \hline
\end{tabular}

The concept originates from purely phenomenological and very simple considerations. The two fragments of the distribution suggest the presence of the substructure. For example, by using this approach, fits of the data at $7 \mathrm{TeV}$ with the shifted-Gamma distribution, in the rapidity windows $|y|<0.5,|y|<1.0$ and $|y|<2.4$, reduces the $\chi^{2} /$ ndf values by a large factor and the distributions become statistically significant with $\mathrm{CL}>0.1 \%$. The results are shown in Table VI. Since the $\alpha_{\text {soft }}$ value is not available, it was input in the distribution and iterated to obtain the best fit. This observation indicates that at higher energies, the contribution of the events with minijets grows. Similar fits when used for data at other energies and rapidity regions also reduce the $\chi^{2} /$ ndf in every case. However, in case of Tsallis distribution, the number of parameters becomes as large as 9 and the fit values of the parameters have very large errors.

Describing the MD as soft and hard components allows one to model, under simple assumptions the new energy

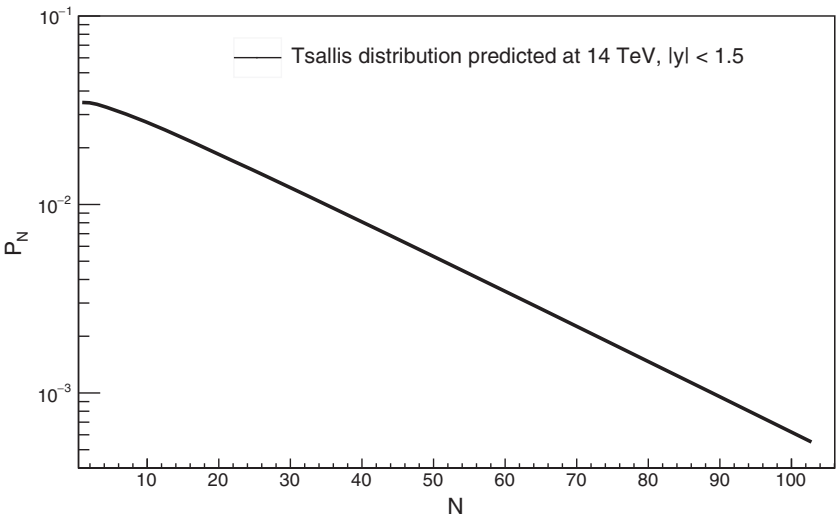

FIG. 6. The multiplicity distribution predicted for $p p$ collisions at $\sqrt{s}=14 \mathrm{TeV}$ in the $|y|<1.5$ window. 
TABLE VI. Fit Parameters with the two-component shifted-Gamma distribution to multiplicity spectra in $p p$ collisions for different rapidity windows and $P_{T}$.

\begin{tabular}{ccccccccc}
\hline \hline$|y|$ & $P_{T}(\mathrm{MeV})$ & $\alpha_{\text {soft }}$ & $\alpha_{1}^{\prime}$ & $\beta_{1}^{\prime}$ & $\alpha_{2}^{\prime}$ & $\beta_{2}^{\prime}$ & $\chi^{2} /$ ndf & $p$ values \\
\hline \multicolumn{7}{c}{$\sqrt{s}=7 \mathrm{TeV}$} \\
0.5 & $>0$ & 0.81 & $2.981 \pm 0.221$ & $0.260 \pm 0.010$ & $7.443 \pm 0.505$ & $1.291 \pm 0.091$ & $11.42 / 35$ & 0.9999 \\
1.0 & $>0$ & 0.77 & $3.702 \pm 0.033$ & $0.161 \pm 0.002$ & $6.985 \pm 0.145$ & $0.695 \pm 0.028$ & $57.91 / 64$ & 0.6904 \\
2.4 & $>500$ & 0.64 & $5.302 \pm 0.101$ & $0.141 \pm 0.003$ & $3.235 \pm 0.030$ & $0.576 \pm 0.023$ & $69.67 / 73$ & 0.5888 \\
\hline \hline
\end{tabular}

domain of $14 \mathrm{TeV}$. While predicting the multiplicity distribution at $14 \mathrm{TeV}$, it remains interesting to determine the dependence of fraction of minijet events as compared to the soft events upon the rapidity window. Present analysis for $7 \mathrm{TeV}$ data shows the expected trend as the minijet fraction of events increasing with rapidity and the transverse momentum. It is expected that when multiplicity distributions measured in full phase space become available, the extrapolations from the lower energy domain will still be valid and very similar. Such an expectation has also been predicted in other works using different approaches $[22,26]$. In case the differences are observed, there is a reason to look for the change in the dynamics of particle production.

\section{CONCLUSION}

A detailed analysis and comparison of the multiplicity spectra of charged particles produced in $p p$ collisions in the restricted rapidity windows at various LHC energies has been done in the framework of the Gamma, the shiftedGamma and the Tsallis distributions. Analysis has also been extended for the particles emitted with $p_{T}>$ $500 \mathrm{MeV}$ in one of the rapidity windows; $|y|<2.4$. The relevance of the comparison is on account of the similar nature of the three distributions. When multiplicity has the Gamma distribution, the average momentum distribution of particles is Tsallis like. For the shifted-Gamma distribution, the average momentum distribution is a possible microcanonical generalization of the Tsallis. The comparison shows that all three distributions reproduce the data in most of the rapidity windows at 0.9 and $2.36 \mathrm{TeV}$. However at $7 \mathrm{TeV}$, all distributions fail and are statistically excluded in the forward region, $|y|<0.5$ and $|y|<1$.0. Interestingly, for the particles emitted with $p_{T}>500 \mathrm{MeV}$, at $\sqrt{s}=7 \mathrm{TeV}$, all the distributions fail at a $\mathrm{CL}<0.10 \%$. The two-component description of the distribution may not suffice. The number of events with minijets may show a structure in the distribution. This might be an indication of the possible dynamical changes in the particle production at such high collision energy. The scale at which hadronization occurs plays an important role in defining the initiation of nonperturbative regime. To find the presence of a possible structure, Zborovský [27] has made a threecomponent analysis, using the negative binomial distributions. However to study the distribution in terms of three-component Tsallis distribution requires a very large statistics and smaller systematic uncertainties, since in the Tsallis distribution fits, the number of fit parameters becomes extremely large. It is claimed in Ref. [27] that the multiplicity characteristics of the third component reveal approximate energy and pseudorapidity invariance, of which a physical explanation represents a challenging problem in high energy multiple particle production.

Overall the Tsallis fits and the shifted-Gamma fits are comparable and much better than the Gamma fits. The value of $q$, a pointer to the nonextensive nature of entropy, measured at each center of mass energy and in different rapidity windows exceeds unity. At a given energy, the value of $q$ decreases with the increase in size of the rapidity window. For collisions at different energies and in the same-size rapidity window, the $q$ value increases with energy, indicating that for collisions at higher energies, the nonextensive behavior of entropy becomes more pronounced. The energy dependence of $q$ is described by the power law. The parametrization as a power law is inspired by the observation that single particle energy distribution obeys a power law behavior [28]. Entropy being determined by the energy fluctuations influences the $q$ values. The $q$ values obtained from our analysis agree very well with the results from another analysis of the data from the ATLAS experiment. Thus the consistent results from two different analyses confirm the $q$-value being weakly dependent on the beam energy and nonextensive nature of the entropy of collisions. For the spectra of particles produced in collider experiments, the underlying theory at a fundamental level is the nonperturbative quantum chromodynamics (QCD) involving long range quark-quark interactions. To effectively describe the complex QCD interactions and hadronization, the Tsallis formalism is specially designed to include self-similar systems and systems with long range interactions. In the Tsallis q-statistics, the nonextensive entropy is an indication of the possible intrinsic, nonstatistical fluctuations. These were identified as the source of the deviations. Such fluctuations are important as possible signals of phase transition(s) taking place in a hadronizing medium [2].

\section{ACKNOWLEDGMENTS}

S. S. is grateful to the Department of Science and Technology, Government of India, for the research fellowship from Grant No. SR/MF/PS-02/2014-PU. 
[1] C. Tsallis, J. Stat. Phys. 52, 479 (1988).

[2] C. E. Agüiar and T. Kodama, Physica (Amsterdam) 320A, 371 (2003).

[3] T. S. Birò, Eur. Phys. J. A 40, 255 (2009).

[4] K. Urmossy and T. S. Birò, Phys. Lett. B 689, 14 (2010).

[5] J. Cleymans, G. Hamar, P. Levai, and S. Wheaton, J. Phys. G 36, 064018 (2009).

[6] C. Beck, Eur. Phys. J. A 40, 267 (2009).

[7] G. Wilk and Z. Wlodarczyk, Eur. Phys. J. A 40, 299 (2009).

[8] I. Bediaga, E. M. F. Curado, and J. M. Miranda, Physica (Amsterdam) 286A, 156 (2000).

[9] S. Sharma, M. Kaur, and Sandeep Kaur, Int. J. Mod. Phys. E 25, 1650041 (2016).

[10] K. Urmossy, G. G. Barnaföldi, and T. S. Birò, Phys. Lett. B 701, 111 (2011).

[11] D. d'Enterria, R. Engel, T. Pierog, S. Ostapchenko, and K. Werner, Astropart. Phys. 35, 98 (2011).

[12] T. Wibig and I. Kurp, J. High Energy Phys. 12 (2003) 039.

[13] Tadeusz Wibig, J. Phys. G 37, 115009 (2010).

[14] M. D. Azmi and J. Cleymans, J. Phys. G 41, 065001 (2014).

[15] J. F. Grosse-Oetringhaus and K. Reygers, J. Phys. G 37, 083001 (2010).
[16] J. Cleymans, J. Phys. Conf. Ser. 455, 012049 (2013).

[17] K. Urmossy, G. G. Barnaföldi, and T. S. Birò, Phys. Lett. B 718, 125 (2012).

[18] J. Cleymans, G. I. Lykasov, A. S. Parvan, A. S. Sorin, O. V. Teryaev, and D. Worku, Phys. Lett. B 723, 351 (2013).

[19] G. Aad et al. (ATLAS Collaboration), New J. Phys. 13, 053033 (2011).

[20] C. Albajar et al. (UA1 Collaboration), Nucl. Phys. B335, 261 (1990).

[21] J. Cleymans, G. I. Lykasov, A. N. Sissakian, A. S. Sorin, and O. V. Teryaev, arXiv:1004.2770; Phys. At. Nucl. 75, 725 (2012).

[22] A. Capella and E. G. Ferreiro, arXiv:1301.3339.

[23] S. Hegyi, Phys. Lett. B 467, 126 (1999).

[24] R. Szwed and G. Wrochna, Z. Phys. C 47, 449 (1990).

[25] V. Khachatryan et al. (CMS Collaboration), J. High Energy Phys. 01 (2011) 079, https://hepdata.net/record/ins879315.

[26] C. Füglesang, Multiparticle Dynamics, edited by A. Giovannini and W. Kittel (World Scientific, Singapore, 1997), p. 193.

[27] I. Zborovský, J. Phys. G 40, 055005 (2013).

[28] M. Gazdzicki and M. I. Gorenstein, Phys. Lett. B 517, 250 (2001). 